\title{
Notas sobre el contexto tectonoestratigráfico de formación de las esmeraldas colombianas
}

\author{
Notes on the tectonostratigraphic context of formation \\ of the Colombian emeralds
}

Roberto Terraza Melo ${ }^{1}$

*Email de correspondencia: rterraza@sgc.gov.co

Recibido: 21 de mayo de 2018

Aceptado: 25 de febrero de 2019

Publicado en línea: 15 de julio de 2019

Doi: 10.32685/0120-1425/boletingeo.45.2019.486

Citación: Terraza M., R. (2019). Notas sobre el contexto tectonoestratigráfico de formación de las esmeraldas colombianas. Boletín Geológico, 45, 37-48. Doi: https://doi.org/10.32685/0120-1425/boletingeo.45.2019.486

\section{RESUMEN}

Las exploraciones geológicas realizadas por el Servicio Geológico Colombiano (SGC) entre los años 2005 y 2008 muestran que las zonas potenciales para exploración y explotación de esmeraldas en el cinturón esmeraldífero occidental (CEOC) están estrechamente relacionadas a las formaciones geológicas Muzo (edad Hauteriviano-Barremiano) y Rosa Blanca (edad Valanginiano), y en el cinturón esmeraldífero oriental (CEOR), a las formaciones Santa Rosa y Chivor, de edad Berriasiano. La mineralización se encuentra asociada a diversas estructuras tectónicas de escala mesoscópica con alteraciones hidrotermales.

Palabras clave: esmeralda, estratigrafía, tectónica, cordillera Oriental, Colombia.

\begin{abstract}
A B S TRACT
The geological explorations carried out by the Colombian Geological Survey (SGC, in Spanish) between 2005 and 2008 show that the potential areas for exploration and exploitation of emeralds in the Western Emerald Belt (CEOC, in Spanish) are closely related to the Muzo (Hauterivian-Barremian age) and Rosa Blanca (Valanginian age) geological formations, and in the Eastern Emerald Belt (CEOR, in Spanish), are associated to the Santa Rosa and Chivor formations (Berriasian age). Mineralization is associated with various mesoscopic tectonic structures with hydrothermal alteration.
\end{abstract}

Key words: Emerald, stratigraphy, tectonic, Cordillera Oriental, Colombia.

1 Servicio Geológico Colombiano. 


\section{INTRODUCCIÓN}

- 1 antiguo Ingeominas, hoy Servicio Geológico Colombiano (SGC), adelantó entre los años 2005 y 2008 la exploración geológica de los cinturones esmeraldíferos de la cordillera Oriental de Colombia, con el propósito de descubrir nuevas áreas potenciales para su explotación. Los estudios geológicos se llevaron a cabo directamente por personal técnico del SGC o mediante convenios de cooperación técnico-científica con la Universidad Industrial de Santander (UIS), y además se contó con la participación de algunos consultores privados (Reyes et al., 2006; Montoya, Terraza, Reyes, Moreno y Fuquen, 2008; Terraza, Montoya, Reyes, Moreno y Fuquen, 2008; Mantilla et al., 2007, 2008; Ingeominas y Mora, 2005).

Se realizó cartografía geológica sistemática a escala 1:25.000, sustentada con bioestratigrafía y estratigrafía detalladas, lo cual permitió generar un nuevo contexto estratigráfico y tectónico de los cinturones esmeraldíferos. A pesar de que ya ha transcurrido una década desde que el SGC oficializó dichos estudios, aún en publicaciones recientes hay un total desconocimiento de los mismos (p. e., Pignatelli et al., 2015).

Con base en el nuevo conocimiento geológico generado en el marco de estas investigaciones, y apoyados por la información geológica disponible de trabajos anteriores, se propuso una serie de criterios geológicos que permitieron reconocer y delimitar nuevas áreas con potencial esmeraldífero, los cuales a su vez sirven para orientar los trabajos mineros relacionados con la exploración y explotación de estos depósitos (Mantilla et al., 2007; Terraza y Montoya, 2011).

El objetivo de estas notas es presentar de manera sencilla y resumida el contexto tectonoestratigráfico de formación de las esmeraldas colombianas localizadas en los denominados cinturones esmeraldiferos oriental y occidental (figura 1).

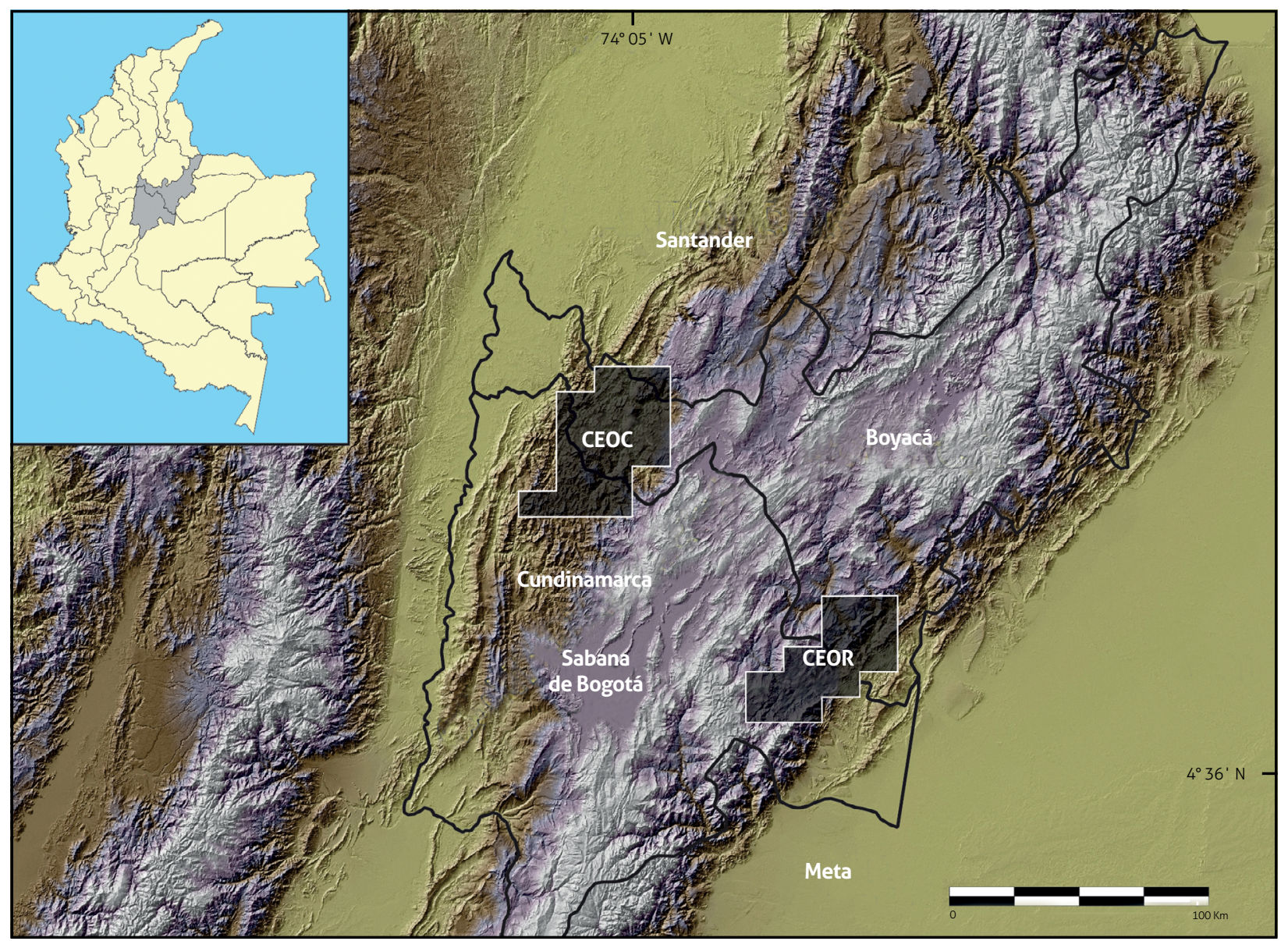

Figura 1. Localización geográfica de los cinturones esmeraldíferos de la cordillera Oriental de Colombia. CEOC = cinturón esmeraldífero occidental, CEOR= cinturón esmeraldífero oriental. Fuente: Terraza y Montoya (2011) 


\section{Generalidades sobre las esmeraldas colombianas}

Las esmeraldas son piedras preciosas que corresponden a una variedad del mineral de color verde conocida como berilo, que pertenece a una clase de minerales denominada ciclosilicatos. Su conspicuo color verde se debe a impurezas en su estructura cristalina debidas a la presencia de pequeñas cantidades de elementos químicos como el cromo y el vanadio; su dureza oscila entre 7,5 y 8 en la escala de Mohs, que va del 1 al 10, donde 1 es el mineral más blando (talco) y 10 el mineral más duro (diamante).

La explotación de esmeraldas en Colombia data de la época precolombina, cuando los muiscas las extraían en el territorio que hoy ocupa el pueblo de Somondoco, para intercambiarlas comercialmente con otros pueblos indígenas. Los españoles continuaron con su explotación en la época colonial, hasta que a principios del siglo XX el Estado colombiano asumió su explotación.

Existen varios modelos geológicos que explican el origen de las esmeraldas que se encuentran en diferentes regiones del mundo, como la actividad magmática, el metamorfismo y los procesos hidrotermales; no obstante, las esmeraldas colombianas son únicas en el mundo por su origen asociado a rocas sedimentarias, que requiere la interacción de varios procesos geológicos, junto a condiciones físico-químicas específicas, sin las cuales ellas no podrían existir.

\section{Contexto estratigráfico de los depósitos}

En Colombia, una zona depocentral relativamente amplia del Cretácico Inferior (abarca las áreas OSsh, SFOS y SFMS alrededor de Bogotá; véase la figura 2) debió jugar un papel muy importante en la circunscripción geográfica de los yacimientos de esmeralda a zonas específicas del país, ya que solo allí se dieron todas las condiciones físico-químicas y los procesos geológicos que posibilitaron su generación. En la actualidad aún se desconocen los límites geográficos exactos de esa área depocentral; sin embargo, con base en los datos geológicos recopilados por el SGC y la UIS durante la exploración geológica realizada en los cinturones esmeraldíferos, se cree que el límite norte se localiza cerca al municipio de Sucre (departamento de Santander), y el límite sur aproximadamente en el municipio de La Palma (departamento de Cundinamarca); de occidente a oriente, esa zona estaría delimitada por los municipios de Puerto Romero y Santa María, en el departamento de Boyacá.

Estos condicionamientos geológicos de las esmeraldas hacen que sus yacimientos se localicen en dos áreas particulares de la cordillera Oriental: una ubicada sobre su borde occidental, en la región de Muzo, Quípama, La Palma, La Victoria, Otanche y San Pablo de Borbur, denominada cinturón esmeraldífero occidental (CEOC), y la otra, sobre su costado oriental, en los municipios de Gachalá, Ubalá, Chivor, Macanal y Santa María, denominada cinturón esmeraldífero oriental (CEOR). Los dos cinturones forman parte de la primitiva área depocentral del Cretácico Inferior. El nombre de cinturón se debe a que las formaciones geológicas que contienen las mineralizaciones de esmeralda configuran geográficamente regiones alargadas en sentido suroccidente-nororiente, de forma similar a un cinturón (véase la figura 1).

Las zonas potenciales para exploración y explotación de esmeraldas en el CEOC se localizan en las formaciones geológicas Muzo (edad Hauteriviano-Barremiano) y Rosa Blanca (edad Valanginiano), y en el CEOR, en las formaciones Santa Rosa y Chivor, de edad Berriasiano. En general, las facies sedimentarias son finogranulares y ricas en materia orgánica, principalmente calcáreas, en el caso de las formaciones Muzo y Rosa Blanca, siliciclásticas en el caso de la Formación Santa Rosa, y calcárea-evaporítica-siliciclástica en el caso de la Formación Chivor. Las rocas corresponden principalmente a arcillolitas y lodolitas calcáreas y micritas de coloración negra muy ricas en materia orgánica. En el caso particular del CEOR, es evidente una estrecha relación entre las mineralizaciones de esmeralda con niveles calcáreo-evaporíticos intercalados, tanto en la Formación Chivor como en la Formación Santa Rosa.

Los cinturones esmeraldíferos están separados entre sí por unos $110 \mathrm{~km}$, y hasta la fecha, en la zona de separación no se han encontrado yacimientos de esmeraldas. En las figuras 3 y 4 se ilustran las sucesiones estratigráficas de cada cinturón esmeraldífero. 


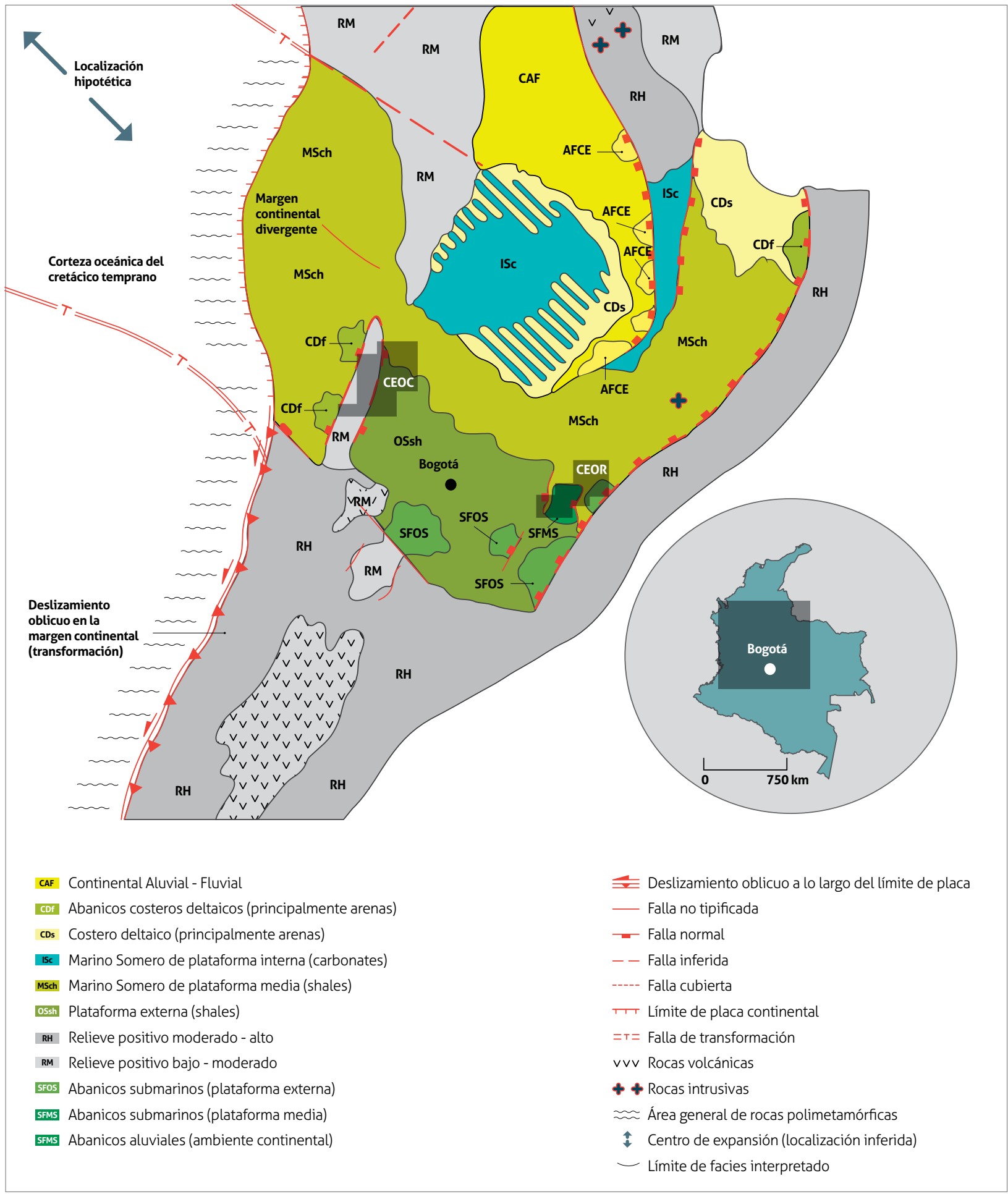

Figura 2. Distribución de facies sedimentarias y armazón tectónico de Colombia a lo largo del Fanenerozoico durante el intervalo de tiempo Berriasiano-Valanginiano. $\mathrm{CEOC}$ = cinturón esmeraldífero occidental; $\mathrm{CEOR}$ = cinturón esmeraldífero oriental.

Fuente: Etayo, Cediel y Cáceres (1997 y 2003) 


\section{Contexto tectónico ReGional de LOS Depósitos}

El CEOC se encuentra fuertemente plegado y fallado. El régimen tectónico es compresivo y transpresivo. Este último se manifiesta por la presencia de fallas inversas con salto transcurrente. El plegamiento es notorio a escala regional y local. Regionalmente se presentan pliegues sinclinales abiertos y frecuentemente con doble inmersión, tanto al NE como al S; a escala de afloramiento hay pliegues anticlinales métricos (de poca longitud), generalmente asimétricos y apretados. Los planos de falla en las zonas con mineralización de esmeralda por lo regular no están mineralizados, y las brechas que normalmente se reconocen en las minas son de origen hidrotermal; no obstante, se pueden confundir con brechas de origen tectónico cuando algunas de las fallas afectan la zona mineralizada. Por los análisis y observaciones de campo realizados, se infiere que las fallas principales son posteriores a las mineralizaciones de esmeralda. La textura finogranular de la mayoría de las unidades litoestratigráficas influye en la deformación de las rocas debido a su comportamiento dúctil, que genera foliación intensa, fallas con poca expresión geomorfológica y, en algunas zonas, replegamiento intenso.

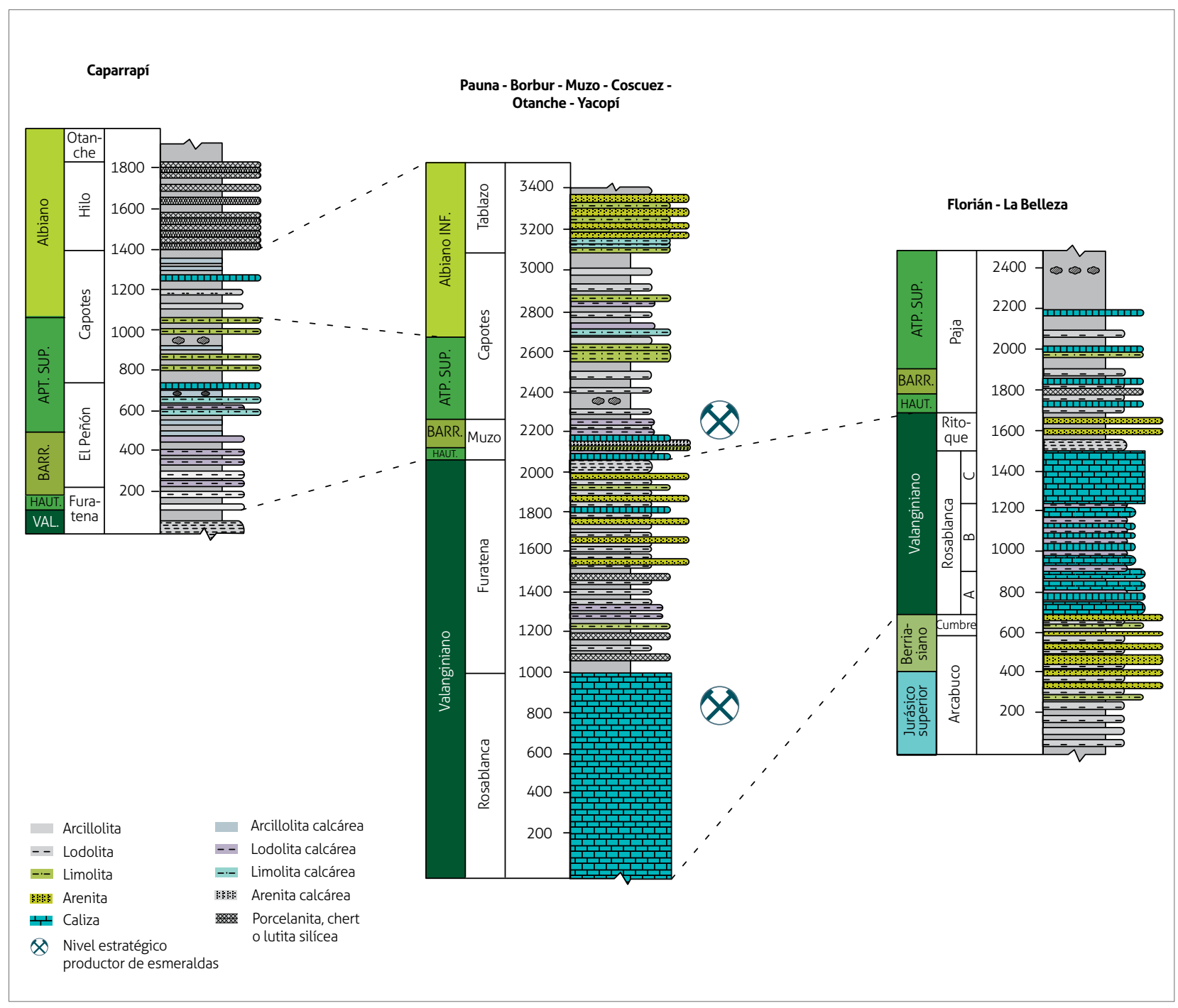

Figura 3. Litoestratigrafía del Cretácico Inferior en el CEOC.

Fuente: Terraza, Montoya y Reyes (2007) 
En el CEOR, el macizo de Quetame atraviesa el cinturón en dirección NE, y en él afloran rocas del Grupo Farallones que conforman el basamento paleozoico sobre el cual se acumularon las rocas del Cretácico. Las rocas cretácicas bordean el macizo, y sus características estructurales dependen de su ubicación con respecto a este basamento paleozoico. $\mathrm{Al}$ oriente, las fallas son estructuras regionales, orientadas en sentido SW-NE, que se extienden por decenas de kilómetros y corresponden a fallas inversas con vergencia oriental, que hacen parte

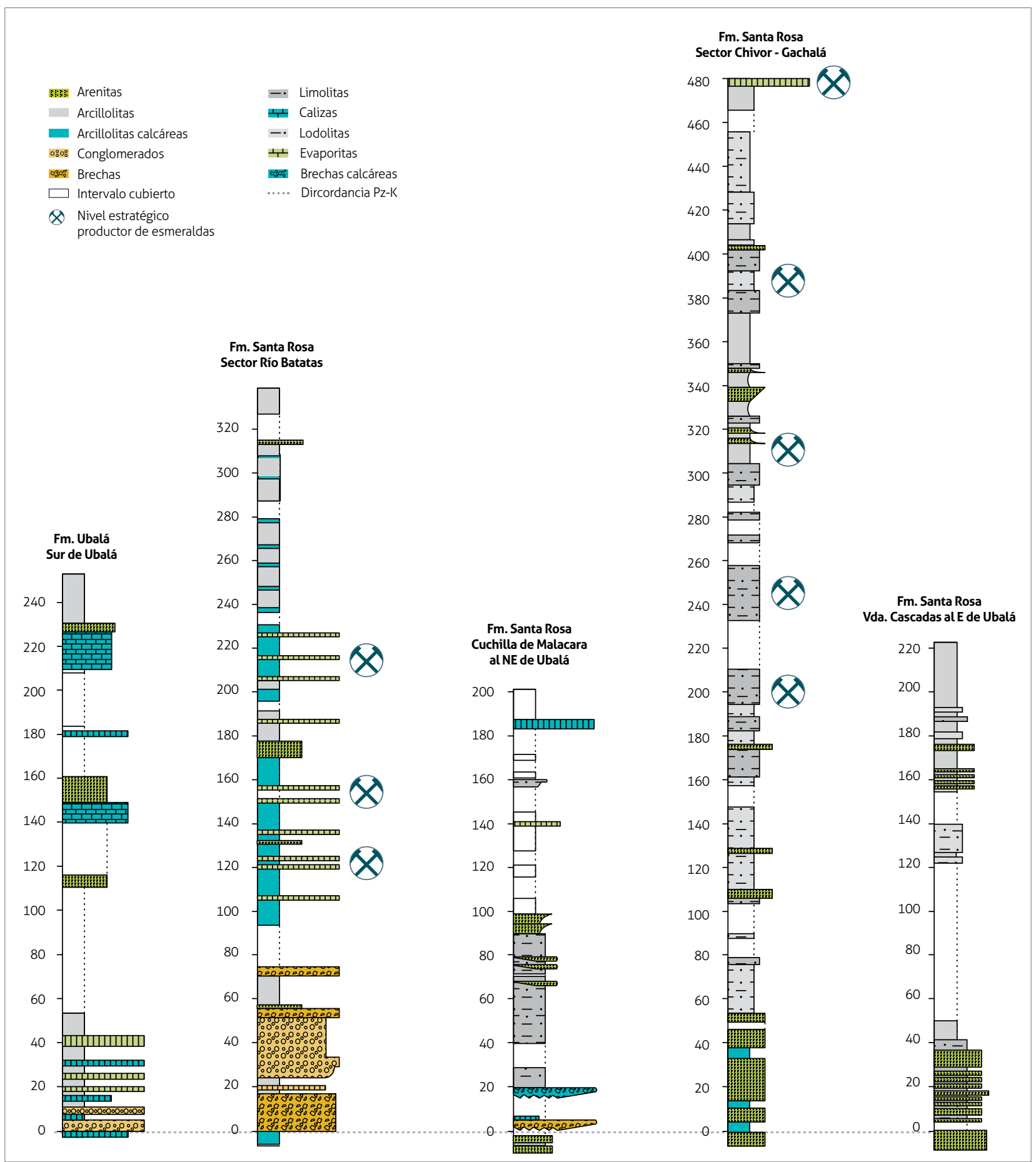

Figura 4. Litoestratigrafía del Cretácico basal en el CEOR.

Fuente: Terraza y Montoya (2011) 
del sistema de fallas del piedemonte llanero. Al occidente se presentan dos tipos de fallas: a) fallas de menor longitud con vergencia al oriente y dirección NE, y que son las responsables del cabalgamiento de rocas paleozoicas sobre cretácicas, y b) otras fallas más regionales, de salto combinado, tanto inverso como transcurrente (dextral o sinestral), con ejes de pliegues oblicuos a su trazo y que repiten secuencia en aquellos sitios en que el segmento de la falla es transpresivo. El plegamiento en las rocas del Cretácico, en general, no es complejo, y se desarrollan pliegues anticlinales y sinclinales amplios y extensos con orientación NE-SW, así como también numerosos pliegues de menor longitud y amplitud. De igual forma, en el CEOR las fallas principales son posteriores a las mineralizaciones de esmeralda.

\section{ReLACIÓN ENTRE DEFORMACIÓN TECTÓNICA, ROCA CAJA Y LA MINERALIZACIÓN DE ESMERALDAS}

Tanto la cartografía geológica regional como los estudios estratigráficos locales (Ingeominas y Mora, 2005; Reyes et al., 2006; Terraza et al., 2008; Montoya et al., 2008) sugieren que las rocas encajantes de las mineralizaciones de esmeraldas se encuentran restringidas, por el contexto paleogeográfico, a ciertas unidades litoestratigráficas del Cretáceo Inferior, como ya se indicó (véanse las figuras 2,3 y 4). La relación establecida con rocas de origen evaporítico (en el caso del CEOR) refuerza la conclusión de Giuliani et al. (1995) y Giuliani, Cheilletz, Rueda y France (1995) en cuanto a un origen a partir de salmueras evaporíticas de buena parte de los fluidos mineralizantes; no obstante, estas condiciones paleoambientales aún no se han podido establecer, en el caso del CEOC. Lo planteado anteriormente sugiere un origen de los fluidos mineralizantes en un sistema cerrado y autóctono, lo que implica migración de fluidos de cortas distancias.

Se plantea una sucesión de eventos tectónicos que dieron origen a las mineralizaciones de esmeraldas (figura 5), lo cual implica que las mineralizaciones están circunscritas a un proceso de plegamiento (Ingeominas y Mora, 2005).

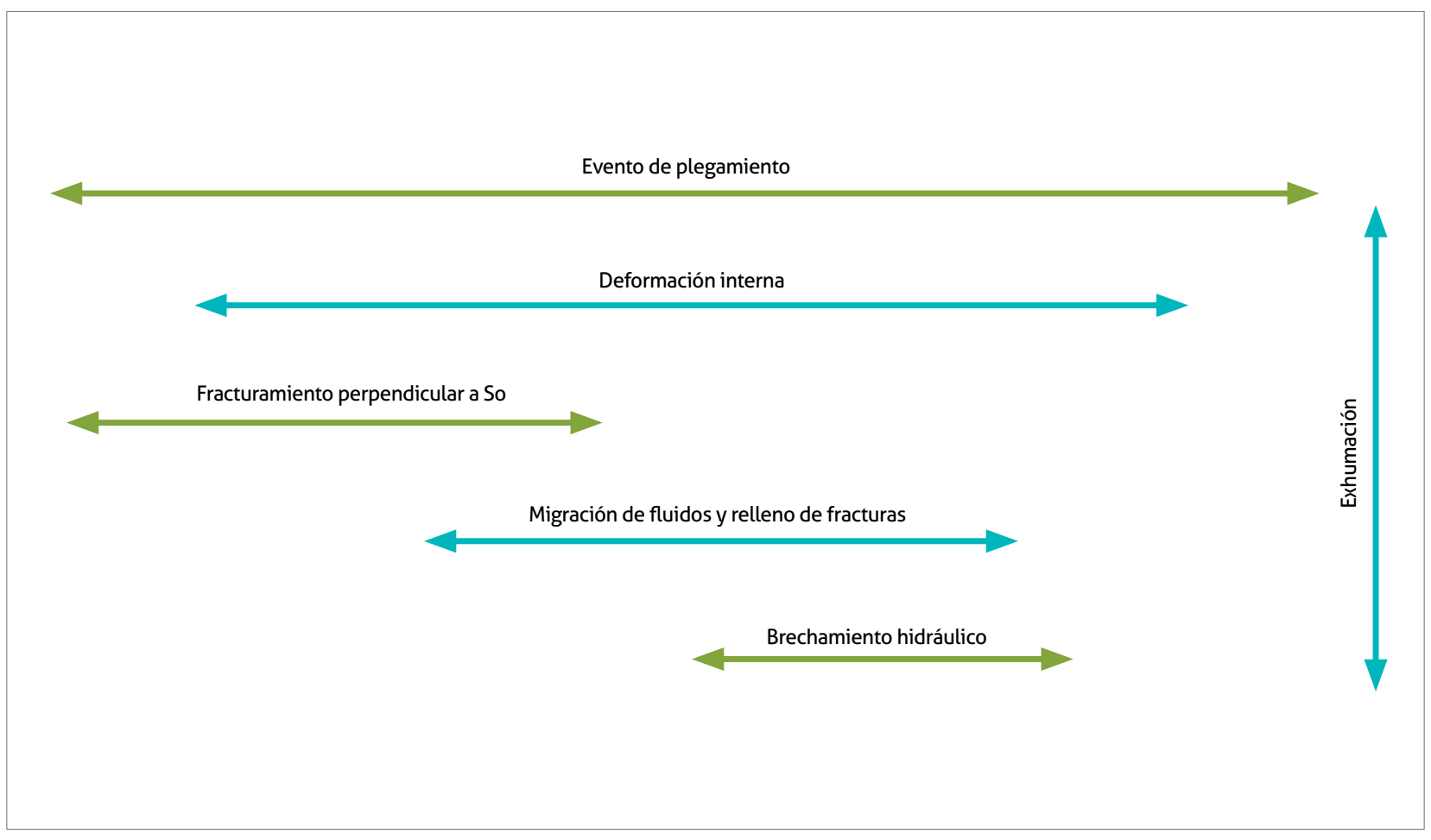

Figura 5. Sucesión de eventos tectónicos generadores de las esmeraldas.

Fuente: Ingeominas y Mora (2005) 
En esta sucesión de eventos se identificaron los siguientes estadios comunes a ambos cinturones esmeraldíferos:

" Fracturas conjugadas que forman ángulos agudos entre sí. Se originan en un estadio de preplegamiento durante el cual, si bien ya existía el régimen de esfuerzos que originó los pliegues, la flexión de las capas asociadas a los mismos aún no se había iniciado, o era de muy bajo ángulo.

" Fracturas paralelas y perpendiculares al eje de pliegues. Se originan durante los estadios iniciales del plegamiento. Este juego de fracturas ortogonales y simétricas respecto al pliegue es un elemento común respecto a otros cinturones plegados y de cabalgamiento.

El hecho de que las fracturas estén o no mineralizadas depende directamente de las unidades que las contienen, entendiéndose en este caso por mineralización cualquier relleno de las fracturas, y no necesariamente la paragénesis de las esmeraldas. En este contexto, las unidades litológicas pelíticas no calcáreas serían las más inadecuadas para contener fracturas mineralizadas; por el contrario, las fisuras de las rocas de las unidades calcáreas serían las más propensas a rellenarse con los fluidos mineralizantes. Este hecho sugiere un régimen de migración de fluidos intraformacional y de cortas distancias en concordancia con lo dicho anteriormente. Por lo tanto, el fracturamiento inicial está relacionado con los estadios iniciales de la deformación tectónica. Dichos estadios iniciales también se asociarían, desde el principio, con una deformación interna penetrativa (foliación tectónica). Una vez estos procesos se inician, las evidencias indican que ante un aumento en la amplitud de los pliegues y de la presión de fluidos se produciría el relleno de las fracturas preexistentes. En este proceso de mineralización, las esmeraldas serían los minerales más internos dentro de un crecimiento sintaxial (es decir, crecen desde las paredes de las venas hacia el interior, algo opuesto a un crecimiento antitaxial, que ocurre desde el interior hacia las paredes).

"En estadios posteriores de deformación, con amplificación aún mayor de los pliegues, las venas mineralizadas extensionales terminan pasivamente en posiciones dentro del pliegue, lo que implica que sufran acortamiento y plegamiento ptigmático. De esta manera, se produce el brechamiento hidráulico ante presión de fluidos finalmente máxima.

"En esta sucesión de eventos, por lo tanto, las mineralizaciones están circunscritas a un proceso de plegamiento.

"El fracturamiento, y sin duda alguna buena parte de las mineralizaciones que rellenan las fracturas, está totalmente ligado a fenómenos flexurales. Aún más, parece ser que ciertas mineralizaciones de calcita se asocian directamente a disolución por presión en las capas directamente adyacentes, donde se genera foliación tectónica y migración directa de fluidos hacia las fracturas circundantes. Sin embargo, no se puede descartar del todo un contexto en el que la mineralización específica de las esmeraldas se relacione con otros procesos, por ejemplo, migración de fluidos hidrotermales externos a la roca encajante. Pese a esto, sean cuales fueren esos procesos adicionales, no fueron anteriores ni posteriores a un proceso de plegamiento y deformación interna: fueron contemporáneos.

" El fracturamiento hidráulico observado (p. e., zona mineralizada de Coscuez-CEOC y río Batatas-CEOR) sugiere que la migración de los fluidos se produjo, probablemente, de modo principal a través de los planos de estratificación. Se observó que las zonas de brechamiento hidráulico no son niveles estratiformes continuos, lo cual implica que dichas zonas no constituyeron vías importantes para la migración de los fluidos mineralizantes. Las fracturas perpendiculares a la estratificación tienen longitudes muy cortas para representar vías de migración representativas. Las zonas principales de falla permanecen como una hipótesis no demostrada; solo en algunos casos puntuales observados sirvieron como eventuales vías de migración de fluidos. En oposición a lo que argumentan Branquet, Laumonier, Cheilletz y Giuliani (1999), no se encontró evidencia alguna de que la migración de fluidos se haya dado principalmente a través de fallas de cabalgamiento. Los casos documentados son aislados y tampoco indican que las zonas de falla o los contactos mecánicos puedan llegar a ser sitios potenciales 
de prospección de esmeraldas (la cercanía o lejanía de las minas de esmeraldas respecto a las fallas son casos más bien fortuitos). Tampoco se observaron casos suficientes como para generalizar sobre la presencia de despegues mineralizados, como lo asumen Cheilletz, Féraud, Giuliani y Rodríguez (1994).

Posteriormente, en etapas más avanzadas de la deformación, áreas como la zona mineralizada de Coscuez en el CEOC sufren un acortamiento aún mayor, probablemente contemporáneo con la fase de mayor exhumación, que bascula las capas a estados subverticales y probablemente rota localmente los ejes de pliegues a lo largo de ejes verticales. Las fracturas previamente formadas también son pasivamente basculadas en este proceso. Finalmente, también se observó que en general hay una sobreimposición de deformación cataclástica tardía, que en ningún caso se observó mineralizada.

Las edades obtenidas por Cheilletz, Féraud, Giuliani y Rodríguez (1991) y Cheilletz et al. (1995), que oscilan entre 32 y $38 \mathrm{Ma}$ en el caso del CEOC, y 62 a 68 Ma en el del CEOR, así como las de Romero, Schultz y Kawashita (2009), de 67 Ma en el caso de los depósitos del CEOC, y de $61 \mathrm{Ma}$ en el del CEOR, concuerdan con estudios regionales (Van der Hammen, 1958; Gómez et al., 2003; Restrepo, Colmenares, Higuera y Mayorga, 2004; Gómez, Jordan, Allmendiger y Cardozo, 2005; Cortés, Angelier y Colleta, 2005) que muestran evidencias de la presencia de deformación y plegamiento desde el Maastrichtiano-Paleoceno y durante el Eoceno. De igual forma, la edad 12,4 $\pm 0,9$ Ma de las esmeraldas del CEOC (Mantilla $e t$ al., 2007) es concordante con procesos de fuerte plegamiento, levantamiento e inicio de erosión en la cordillera Oriental durante el Mioceno Medio (Van der Hammen, 1958; Cooper et al., 1995).

Los estudios de microsonda y de inclusiones fluidas de Cheilletz et al. (1994) sirvieron de base para sustentar la hipótesis de que las mineralizaciones se producen antes de los procesos mayores de exhumación de la cordillera Oriental, ya que el inicio de la exhumación puede ser un factor capaz de detener los procesos mineralizantes, dadas las condiciones de presión y temperatura propuestas por los citados investigadores.
Los mineros extraen las esmeraldas de las "estructuras hidrotermales", que se originan cuando los fluidos mineralizantes transportan los elementos o sustancias que forman la esmeralda, y "penetran" en la roca sedimentaria sometida a deformación tectónica (plegamiento, deformación interna y fracturamiento), lo que hace que la roca se rompa, y en esas fracturas, debido a procesos de cristalización, se forman distintos minerales, incluida la esmeralda; estas estructuras se conocen como "venas", "brechas hidráulicas" o "zonas o bandas albitizadas" (figura 6). Comúnmente, la esmeralda está acompañada de otros minerales que son típicos de los yacimientos esmeraldíferos, como la albita, la calcita, carbonatos de elementos de las tierras raras (como la parisita), fluorita, barita y algunos sulfuros (p. e., pirita y calcopirita). Los mineros tradicionales, que normalmente no tienen el conocimiento geológico de los yacimientos, han explotado este recurso de manera empírica desde hace muchos años, porque aprendieron a reconocer tanto los minerales asociados a la esmeralda como las diferentes estructuras hidrotermales presentes en la roca. Su extracción generalmente es subterránea, mediante la excavación de túneles con ramales en distintas direcciones, literalmente persiguiendo las estructuras hidrotermales mencionadas, aunque en otras minas su explotación puede ser superficial.

Como se puede ver, la geología de los yacimientos de esmeraldas en Colombia es bastante compleja. La exploración de cualquier recurso mineral (hidrocarburos, agua subterránea, carbón, esmeraldas u otro mineral) implica un conocimiento geológico detallado del territorio, tanto a nivel de superficie como del subsuelo, que se logra mediante diversas disciplinas de las geociencias, como la cartografía geológica, estratigrafía, geoquímica, geofísica, estudios satelitales, paleontología, geocronología y otras más. La cantidad de recurso mineral disponible dependerá del grado de conocimiento geológico que se tenga y del avance tecnológico de la minería, pues no es lo mismo extraerlo con métodos de hace quinientos años, cuando nuestros ancestros extraían las esmeraldas de forma rudimentaria sin ningún conocimiento geológico, a como lo hacemos hoy en día. No obstante, aún nos falta mucho conocimiento sobre la geología de nuestros yacimientos esmeraldíferos. 

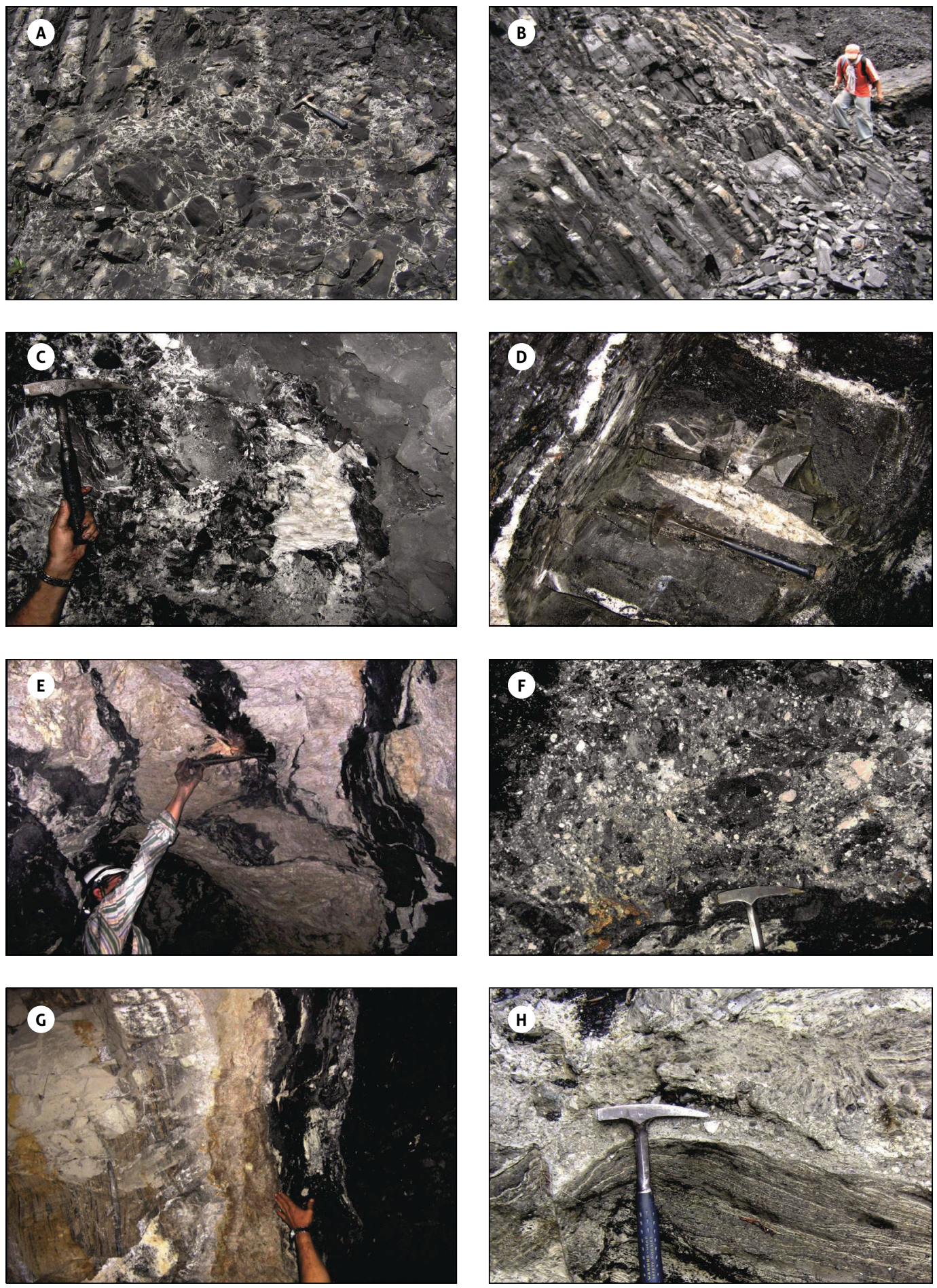

Figura 6. Estructuras hidrotermales típicas de las zonas con mineralización de esmeraldas en Colombia. A) Brecha hidráulica de fragmentos de arcillolita calcárea carbonosa cementados por calcita. B) Venas de calcita paralelas a la estratificación. C) Lodolita calcárea carbonosa con venas de calcita-dolomita de aspecto brechoide. D) Venas de calcita lenticulares transversales a la estratificación. E) Lodolita carbonosa con bandeamientos albitíticos. F) Brecha de clastos de lodolita cementados con calcita y dolomita. G y H) Nivel albitizado con pirita, calcita y dolomita. Localización geográfica: A y B) minas de Coscuez, Formación Muzo; C y E) minas de Muzo, Formación Muzo; D) minas de Peñas Blancas, Formación Rosa Blanca; F) minas de Gachalá, Formación Santa Rosa; G y H) minas de Chivor, Formación Chivor.

Fuente: Terraza y Montoya (2011) 


\section{Conclusiones}

Las zonas potenciales para exploración y explotación de esmeraldas, en el CEOC están restringidas a las formaciones geológicas Muzo (edad Hauteriviano-Barremiano) y Rosa Blanca (edad Valanginiano), y en el CEOR, a las formaciones Santa Rosa y Chivor, de edad Berriasiano.

La sucesión de eventos geológicos más importantes que dieron origen a las esmeraldas fueron los siguientes:

1. Acumulación de espesas secuencias de sedimentos finogranulares, ricos en materia orgánica, carbonatos y evaporitas, en varios episodios del Cretácico Temprano de Colombia, como en el piso Berriasiano (135-131 Ma), Valanginiano (131-123 Ma) y Hauteriviano-Barremiano (123-117 Ma). Esta acumulación de sedimentos ocurrió en áreas depocentrales o de mayor subsidencia de la antigua cuenca sedimentaria cretácica colombiana (véase la figura 2).

2. Enterramiento de los sedimentos a varios kilómetros de profundidad, que permitió que la roca alcanzara alta diagénesis y bajo grado de metamorfismo (temperaturas $>100-200{ }^{\circ} \mathrm{C}$ y presiones $>1 \mathrm{kbar}$ ).

3. Sucesión de eventos tectónicos que afectaron a la roca sedimentaria como plegamiento, deformación interna, fracturamiento, migración del fluido mineralizante de la esmeralda, relleno de fracturas y brechamiento hidráulico; se han identificado varios eventos tectónicos asociados a las esmeraldas que tienen edades geológicas que van desde 62 a $68 \mathrm{Ma}$ (Paleoceno Tardío-Maastrichtiano), 32 a $38 \mathrm{Ma}$ (Eoceno Tardío) y $12 \mathrm{Ma}$ (Mioceno Tardío).

4. Exhumación de la roca con los yacimientos de esmeralda posterior a los $12 \mathrm{Ma}$ (en este caso particular se hace referencia al levantamiento e inicio de los procesos erosivos en la cordillera Oriental, sitio en el cual se localizan los yacimientos esmeraldíferos colombianos).

5. Las mineralizaciones de esmeraldas se encuentran asociadas a diferentes estructuras tectónicas con alteración hidrotermal, como venas, brechas hidráulicas o zonas o bandas albitizadas.

\section{Agradecimientos}

A los revisores anónimos del manuscrito por sus comentarios y sugerencias.

\section{REFERENCIAS BIBLIOGRÁFICAS}

Branquet, Y., Laumonier, B., Cheilletz, A. y Giuliani, G. (1999). Emeralds in the Eastern Cordillera of Colombia: Two tectonic settings for one mineralization. Geology, 27 (7), 597-600.

Cheilletz, A., Féraud, G., Giuliani, G. y Rodríguez, C. (1991). ${ }^{40} \mathrm{Ar} /{ }^{39} \mathrm{Ar}$ laser-probe dating of the Colombian emerald deposits: Metallogenic implications. En M. Pagel y J. L. Leroy (eds.), Source, Transport and Deposition of Metals, Proceedings of the 25 years SGA Anniversary Meeting, Nancy, 30 August-3 September 1991, Rotterdam (A. A. Balkema), 373-376.

Cheilletz, A., Féraud, G., Giuliani, G. y Rodríguez, C. (1994). Time-pressure and temperature constraints on the formation of Colombian Emeralds: $\mathrm{An}{ }^{40} \mathrm{Ar} /$ $\mathrm{Ar}^{39}$ laser microprobe and fluid inclusion study. Economic Geology, 89 (2), 361-380.

Cheilletz, A., Giuliani, G., Zimmermann, J. L., Ribeiro Althoff, A. M., Féraud, G. y Rueda, F. (1995). Ages, geochemical signatures and origin of Brazilian and Colombian emerald deposits: A magmatic versus sedimentary model. En J. Pasava, B. Kribek y K. Zák (eds.), Mineral Deposits: From their origin to their environmental impacts. Proc 3rd Biennial SGA Mtg., Prague, AA Balkema, Rotterdam, 569-572.

Cooper, M. A., Addison, F. T., Álvarez, R., Coral, M., Graham, R. H., Hayward, A. B. et al. (1995). Basin development and tectonic history of the Llanos Basin, Eastern Cordillera, and Middle Magdalena Valley, Colombia. American Association of Petroleum Geologists Bulletin, 79 (10), 1421-1442.

Cortés, M., Angelier, J. y Colleta, B. (2005). Paleostress evolution of the northern Andes (Eastern Cordillera of Colombia): Implications on plate kinematics of the South Caribean region. Tectonics, 24 (1), 1-27. Doi: https://doi.org/10.1029/2003TC001551.

Etayo, F., Cediel, F. y Cáceres, C. (1997). Mapa de distribución de facies y ámbito tectónico a través del Fanerozoico de Colombia. Escala 1:1'500.000. Bogotá: Ingeominas, Geotec Ltda.

Etayo, F., Cediel, F. y Cáceres, C. (2003). Mapa de distribución de facies y ámbito tectónico a través del Fanerozoico de Colombia. Escala 1:1'500.000. Bogotá: Ingeominas, Geotec Ltda. 
Giuliani, G., Cheilletz, A., Arboleda, C., Carrillo, V., Rueda, F. y Baker, J. H. (1995). An evaporitic origin of the parent brines of Colombian emeralds: Fluid inclusion and sulphur isotope evidence. European Journal of Mineralogy, 7 (1), 151-165. Doi: https://doi.org/10.1127/ejm/7/1/0151

Giuliani, G., Cheilletz, A., Rueda, F. y France, L. C. (1995). The genesis of Colombian emerald deposits: An unique example of beryllium mineralization developed in a black shale environment. En J. Pasava, B. Kribek y K. Zák (eds.), Mineral deposits: From their origin to their environmental impacts, Proc 3rd Biennial SGA Mtg., Prague, AA Balkema, Rotterdam, 943946.

Gómez, E., Jordan, T. E., Allmendiger, R. W., Hegarty, K., Kelley, S. y Heizler, M. (2003). Controls on architecture of the Late Cretaceous to Cenozoic Southern Middle Magdalena Valley Basin, Colombia. Geological Society of America Bulletin, 115 (2), 131-147. Doi: https://doi. org/10.1130/0016-7606(2003)1150131COAOTL>2.0. $\mathrm{CO} ; 2$.

Gómez, E., Jordan, T. E., Allmendiger, R. W. y Cardozo, N. (2005). Development of the Colombian foreland-system basin as a consequence of the diachronous exhumation of the northern Andes. Geological Society of America Bulletin, 117 (9-10), 1272-1292. Doi: https://doi.org/10.1130/B25456.1

Ingeominas y Mora, A. (2005). Levantamiento de Información estratigráfica y estructural de los cinturones esmeraldíferos de la cordillera Oriental. Informe técnico, contrato de prestación de servicios BTA-013. Bogotá: Ingeominas.

Mantilla, L. C., Silva, A., Serrano, J. J., Conde, J., Gómez, C., Ramírez, J. C. et al. (2007). Investigación petrográfica y geoquímica de las sedimentitas del Cretácico Inferior (K1) y sus manifestaciones hidrotermales asociadas; planchas 169, 170, 189, 190 (cordillera Oriental): implicaciones en la búsqueda de esmeraldas. Acuerdo específico 1 de 2005. Bogotá: Ingeominas-Universidad Industrial de Santander.

Mantilla, L. C., Silva, A., Conde, J., Gaviria, J. A., Gallo, F., Torres, D. A. et al. (2008). Estudio de los procesos de interacción fluido-roca en el cinturón esmeraldífero oriental (cordillera Oriental, Colombia) y su importancia en la exploración de nuevos yacimientos hidroter- males. Acuerdo específico 2 de 2006. Bogotá: Ingeominas-Universidad Industrial de Santander.

Montoya, D., Terraza, R., Reyes, G., Moreno, G. y Fuquen, J. (2008). Geología del cinturón esmeraldífero oriental, planchas 210, 228 y 229 (mapa). Escala 1:100.000. Bogotá: Ingeominas.

Pignatelli, I., Giuliani, G., Ohnenstetter, D., Agrosì, G., Mathieu, S., Morlot, C. et al. (2015). Colombian trapiche emeralds: Recent advances in understanding their formation. Gems \& Gemology, 51 (3), 222-259.

Romero, F. H., Schultz Güttler, R. y Kawashita, K. (2000). Geoquímica del rubidio-estroncio y edad de las esmeraldas colombianas. Geología Colombiana, 25, 221-229.

Reyes, G., Montoya, D., Terraza, R., Fúquen, J., Mayorga, M. y Gaona, T. (2006). Geología del cinturón esmeraldífero occidental, planchas 169, 170, 189 y 190 (mapa e informe). Escala 1:100.000. Bogotá: Ingeominas.

Restrepo, P. A., Colmenares, F., Higuera, C. y Mayorga, M. (2004). A fold-and-thrust belt along the western flank of the Eastern Cordillera of Colombia: Style, kinematics, and timing constraints derived from seismic data and detailed surface mapping. En K. R. McKlay (ed.), Thrust tectonics and hydrocarbon systems, vol. 82 Tulsa: American Association of Petroleum Geologists. Doi: https://doi.org/10.1306/M82813C31.

Terraza, R. y Montoya, D. (2011). Las esmeraldas de Colombia en su ámbito geológico: excursión geológica a los cinturones esmeraldíferos de la cordillera Oriental de Colombia en el marco del XIV Congreso Latinoamericano de Geología del 29 de agosto al 2 de septiembre de 2011.

Terraza, R., Montoya, D. y Reyes, G. (2007). El Cretácico Inferior en el cinturón esmeraldífero occidental. Memorias XI Congreso Colombiano de Geología, Bucaramanga, agosto 14 al 17 de 2007.

Terraza, R., Montoya, D., Reyes, G., Moreno, G. y Fuquen, J. (2008). Geología del cinturón esmeraldífero oriental, planchas 210, 228 y 229 (informe). Bogotá: Ingeominas.

Van der Hammen, T. (1958). Estratigrafía del Terciario y Maastrichtiano continentales y tectogénesis de los Andes colombianos. Boletín Geológico, 6 (1-3), 67128. 